tolerance, which is also shared by the benzodiazepine agents, a review of published work in 1967 showed that AZM was equal in efficacy to ethosuximide and had a lower incidence of side effects (Millichap JG, Aymat F. Treatment and prognosis of petit mal epilepsy. Pediatr Clin N Am $1967 ; 14: 905)$. At least as an adjunct therapy, the drug deserves wider recognition and confirmation of efficacy by controlled studies.

\title{
SIDE EFFECTS OF ANTICONVULSANT MONOTHERAPY
}

The severity and nature of side effects and doses and plasma levels of phenobarbital (PB), primidone (PRM), phenytoin (PHT), carbamazepine (CBZ), and valproate (VPA) were examined in 392 pediatric outpatients at the Division of Paediatric Neurology, National Hospital "Marques de Valdecilla", University of Cantabria, Santander, Spain. Side effects occurred in 50\%, necessitating changes in medication in $18 \%$ and withdrawal of drug in $7 \%$. The incidence of side effects was highest with PHT (71\%) and lowest with PRM (29\%). Serious side effects requiring drug withdrawal occurred with PHT (10\%), VPA (8\%), and PRM (8\%), and less frequently with $\mathrm{PB}(4 \%)$ and $\mathrm{CBZ}$ (3\%). The best tolerated drug was CBZ, and the least tolerated was PHT. Behavioral side effects were noted most commonly with PB (60\%), neurological abnormalities such as ataxia and nystagmus with PHT (22\%), digestive tract disorders with VPA (28\%), and gingival hyperplasia and hirsutism with PHT (588). The side effects that most often necessitated changes in treatment were behavioral disorders, especially excitement and hyperactivity, with PB and PRM, hirsutism and gingival hyperplasia with PHT, restless sleep and vomiting with CBZ, and digestive disorders with VPA. Behavioral disorders produced by PB and PRM disappeared in half of the patients if the dosage of the drug was increased. (Herranz JL et al. Clinical side effects of phenobarbital, primidone, phenytoin, carbamazepine, and valproate during monotherapy in children. Epilepsia Nov/Dec 1988; 29:794-804).

COMMENT. A collaborative group for epidemiology of epilepsy reports a $42 \%$ incidence of adverse reactions to antiepileptic drugs in 355 patients followed for an average of 11 months in 15 university and hospital centers in Italy (Beghi $\mathrm{E}$ et al. Institute for Pharmacological Research "Mario Negri," Milan. Epilepsia Nov/Dec 1988;29:787). Clinical judgment provided the most valid basis for the evaluation of drug toxicity, and "toxic" plasma drug levels were not correlated with adverse reactions. Plasma levels were within normal limits in $78 \%$ of cases with and in 818 of cases without adverse drug reactions. Of 31 patients with "abnormal" plasma levels, only 1 had an adverse drug reaction. The authors stress the importance of reporting adverse drug reactions as a means of improving the quality of care for the epileptic in routine clinical practice. Physicians are sometimes reluctant to get involved, fearing legal repercussions or lengthy and tedious questionnaires from drug companies. Perhaps a system permitting anonymity might encourage more active physician participation. 
Additional, more sensitive, methods of monitoring drug treatment should expand the concept of "intolerable" side effects to include subtle psychological and behavioral effects. (Vining EPG et al. Pediatrics 1987; 80:165).

\section{TERATOGENIC EFFECTS OF DIAZEPAM}

Eight children exposed in utero to benzodiazepines, diazepam or oxazepam, and having dysmorphic characteristics resembling the fetal alcohol syndrome (FAS) are reported from the Department of Pediatrics, Gothenburg University, Sweden. The most common craniofacial abnormalities were slanted eyes, epicantic folds, short, uptilted nose, flat upper lip, and hypoplastic mandible. All were hypotonic from birth and had neonatal drug withdrawal symptoms of opisthotonus and convulsions. Low Apgar scores were recorded in 6 , mainly because of apnea, and 5 were resuscitated. Rooting and sucking reflexes were absent and feeding difficulties prominent. Gross motor disability in all children, 2 having spastic hemiparesis, was seen during early life, and fine motor incoordination and tremor were common in older children. One infant who died had neuronal migration defects and heterotopias. (Laegreid L et al. Teratogenic effects of benzodiazepine use during pregnancy. $\mathrm{J}$ Pediatr Jan $1989 ; \underline{114}: 126-31)$.

COMMENT. Alcohol ingestion in these mothers was excluded. The fetal diazepam syndrome (FDS) differed from the FAS in a greater focal involvement of cranial nerves, a sullen and expressionless facies, and more frequent occurrence of low Apgar scores, apneic spells, delayed motor development, and neonatal hypotonia. There have been numerous previous reports of the "floppy infant syndrome" in babies born to women treated with long-term diazepam during pregnancy. Bilateral opercular polymicrogyria and grey matter heterotopias found at autopsy of 2 cases of Foix-Chavany-Marie syndrome (faciopharyngealglossomasticatory diplegia) (Becker PS et al. Ann Neurol Jan 1989;25:90) might sometimes be explained by diazepam teratogenicity. This syndrome is rare and usually follows stroke and vascular infarction in adults. There are very few reports of the pathology in developmental varieties of the syndrome.

\section{MOVEMENT OF $\mathrm{X}$ CHROMOSOME IN EPILEPTIC CORTEX}

Chronic uncontrolled seizure activity was linked to specific positional changes of the $x$ chromosome in neurons of cortical seizure foci from both male and female patients at Yale University School of Medicine, New Haven, Connecticut. In normal female neurons, $X$ homologs were typically either on the nucleolus or on the nuclear membrane, whereas one or both $\mathrm{X}$ chromosome signals were moved interiorly in seizure foci of 2 representative female patients. There was a gradient of $x$ positional changes spreading from the seizure focus toward normal surrounding cortex. In seizure foci there was a decrease of $25 \%$ in membrane plus nucleolar signal neurons compared to more normal specimens, and a concomitant increase of $27 \%$ of 